Review Article

\title{
P2X7 Receptor as a Key Player in Oxidative Stress-Driven Cell Fate in Nonalcoholic Steatohepatitis
}

\author{
Saurabh Chatterjee and Suvarthi Das \\ Environmental Health and Disease Laboratory, Department of Environmental Health Sciences, University of South Carolina, \\ Columbia, SC 29208, USA \\ Correspondence should be addressed to Saurabh Chatterjee; schatt@mailbox.sc.edu
}

Received 31 December 2014; Accepted 17 February 2015

Academic Editor: Peter Backx

Copyright (C) 2015 S. Chatterjee and S. Das. This is an open access article distributed under the Creative Commons Attribution License, which permits unrestricted use, distribution, and reproduction in any medium, provided the original work is properly cited.

\begin{abstract}
Incidences of nonalcoholic fatty liver disease parallels increase in the global obesity epidemic. NAFLD has been shown to be associated with risks of cardiometabolic disorders and kidney disturbances. It is accompanied by insulin and leptin resistance that complicate the diagnosis and treatment of this public health menace. Though significant research is underway for understanding the molecular mechanisms of NAFLD and its subsequent inflammatory and fibrotic manifestations like nonalcoholic steatohepatitis, the role of purinergic receptors has been unclear. It is increasingly being recognized that damage associated molecular patterns like NAD and ATP that are released from injured cells via hepatocellular injury either by oxidative stress or lipotoxicity from steatosis activate the purinergic receptor. Based on evidence from inflammatory responses in the airways and vasculature and autoimmune complications in humans and rodents, it is beyond doubt that hepatocellular inflammation such as that seen in NASH can result from the activation of purinergic receptors. This event can result in the formation of inflammasomes and can be an important pathway for the progression of NASH. The present review evaluates the current knowledge of the role of oxidative stress and its signaling via P2X7 receptors in hepatocellular injury that might contribute to the NASH pathophysiology.
\end{abstract}

\section{Introduction}

The "Global Society" is driving us towards a global epidemic of obesity, type 2 diabetes mellitus (T2DM), and metabolic syndrome (MS), with each passing day. Insulin resistance is the most closely associated pathophysiological hallmark [1$3]$, whereas nonalcoholic fatty liver disease (NAFLD) is the hepatic manifestation of the metabolic syndrome [4]. The estimated worldwide prevalence of NAFLD is $6.3 \%-33 \%$ with a median of $20 \%$ in the general population. However, in the presence of obesity and T2DM, the prevalence of NAFLD increases to about 75\% [5-8]. NAFLD will be the most important chronic liver disease within a few years posing a grave challenge for the gastroenterologists and the hepatologists worldwide $[9,10]$. The complex spectrum of NAFLD constitutes of benign steatosis, more severe alterations like NASH (nonalcoholic steatohepatitis), cirrhosis, and sometimes hepatocellular carcinoma $[10,11]$. The pathogenesis proceeds from accumulation of fat in the liver followed by liver injury, inflammation, and fibrosis and then scarring of liver, till the scar tissue replaces the liver cells, giving rise to the cirrhotic phase, primarily resulting from an aberrant tissue repair process [11]. Cirrhosis is irreversible and if untreated; the cirrhotic liver can progress to hepatocellular carcinoma. The mechanisms that account for disease progression in NAFLD are still poorly understood. Yet a crucial stage that might be treated as an early indicator for the initiation of NASH from benign steatotic and usually asymptomatic liver might be that of sinusoidal endothelial injury [12].

\section{The Concept of 2nd Hit and Multiple Hits: Roles of Oxidative Stress and Proinflammatory Signaling Pathways}

The specific stages or the mechanistic pathway for the progression of fatty liver to NASH is based on theories till date. The "two-hit" hypothesis put forward by Day and James 
in 1998, recognizes lipid accumulation in the hepatocytes as the first hit, characterized by unaltered lipid metabolism in the liver [13]. The hypothesis evolved from the study which showed that ob/ob mice and fa/fa rats were exquisitely vulnerable to LPS-induced liver injury and concluded further that fatty livers were susceptible to liver injury from secondary stressors [14]. Later study from Yamaguchi et al. showed that hepatocyte triglyceride accumulation per se was not detrimental but inhibition of triglyceride synthesis exacerbated liver injury [15]. Rather, triglyceride accumulation was a protective mechanism that buffered hepatocytes from toxicity of nonesterified fatty acids that flooded the liver following insulin resistance [16]. Obesity-induced insulin resistance which likely arises from the effects of TNF- $\alpha$ has been observed to be a key pathogenic factor for the development of hepatic steatosis [1,17-19]. One would argue that hepatic steatosis might be contributing to the underlying condition of hepatocyte stress based on evidence that polymorphisms of PNPLA3 are a major risk factor for NASH and cirrhosis. The 2nd of the two hits could be due to (i) oxidative stress, (ii) proinflammatory cytokines and adipokines, (iii) mitochondrial dysfunction, or (iv) endoplasmic reticulum stress. Any one of these 2nd hits could lead to hepatocyte injury, inflammation, and then fibrosis. In 2010, a more holistic model of "multiple parallel hits" was proposed by Tilg and Moschen wherein many parallel hits derived from the gut and/or the adipose tissue that may promote liver inflammation were identified [20]. Endoplasmic reticulum stress and related signaling networks, (adipo)cytokines, and innate immunity are emerging as central pathways that regulate key features of NASH.

\section{Oxidative Stress in NASH Progression}

Progression from NAFLD to NASH is multifactorial and there have been implications of oxidative stress as a crucial trigger for NASH, although a more specific cause-effect relationship between oxidative stress and NASH has not yet been established [21-23]. Increased levels of reactive oxygen species and lipid oxidation products and decreased levels of antioxidant enzymes such as superoxide dismutase (SOD) and catalase and antioxidant compounds such as glutathione have been observed in patients of NAFLD/NASH compared with those observed in the healthy subjects. Various kinds of oxidants have been reported to cause lipid oxidation: enzymatic oxidants namely cytochrome P450, lipooxygenase, cyclooxygenase and so forth, and/or nonenzymatic oxidants such as free radicals, hypochlorite, or singlet oxygen might have singular or synergistic effect in causing oxidative stress $[21,24]$. CYP2E1, an isoform of cytochrome P450, expressed in the liver, has emerged as an important cause of ROS overproduction, as shown in both rodent and human models $[25,26]$. Lipid oxidation products render damage in multiple ways: they are cytotoxic, cause damage to proteins and DNA, and often act as ligands or antagonists for various receptors and thus modulate inflammation $[21,27,28]$. The levels of oxidative stress markers such as 4-hydroxynonenal and 4hydroxydeoxyguanosine correlate well with the severity of necroinflammation and subsequent fibrosis [29-31]. In this scenario, recent evidence indicates that lipid peroxidation products originating from the oxidation of phospholipids can act as damage associated molecular patterns (DAMPs) and promote inflammation through the interaction with soluble and cell-associated pattern recognition receptors [32, 33]. Mitochondria are the most important cellular source of ROS, and mitochondrial dysfunction might therefore be a common denominator in the pathological mechanisms of NASH [34]. Although the mechanisms of mitochondrial dysfunction are not clearly understood, emerging data suggest that ROS, lipid peroxidation products are involved in the second hit namely oxidative stress, which induces the progression of simple steatosis to NASH. Furthermore, ROS induce the directional migration of resident hepatic profibrogenic cells, resulting in liver fibrosis [35-37]. Considering oxidative stress as a second hit might be a feasible starting point, but it would be reasonable to assume that multiple, rather than single, prooxidative intracellular and extracellular triggers act in conjunction promoting oxidative stress that drives the development of NASH [34]. Recent reports have shown the involvement of peroxynitrite as an important reactive species in NASH pathogenesis. Chatterjee et al. have shown that peroxynitrite from NADPH oxidase activates Kupffer cells and causes inflammation in the steatotic liver [38]. Unpublished reports from our laboratory have shown the involvement of peroxynitrite in causing toll like receptor 4 trafficking into the lipid rafts, a significant event in TLR4 activation and signaling in NASH. The trafficking process was attenuated by peroxynitrite scavenger phenyl boronic acid (FBA) that has been reported to be specifically reacting with peroxynitrite [39]. Though the involvement of NADPH oxidases have been shown in NASH pathophysiology, especially in fibrosis related complications, the molecular basis of NADPH oxidase mediated ROS generation is now beginning to evolve following the use of species-specific probes like FBA and a better understanding of the free radical chemistry that might trigger the generation of reactive oxygen and nitrogen species in NASH. However, it remains to be seen whether the sequence of events following hepatocyte necrosis via lipotoxicity is causing innate immune responses and subsequent oxidative stress or vice versa.

\section{Oxidative Stress and Hepatocyte Degeneration/Necrosis}

ROS generated by CYP2E1 and other sources might induce damage in two ways: first, as stated before by direct modulation of cellular functions by modification/peroxidation of lipids, proteins, and DNA [40,41]. The second mechanism could be the activation of the cell-death signaling pathways. This activation might either be from ROS or reactive byproducts of lipid peroxidation, 4-HNE in particular, which has been shown to activate JNK [41-44]. Lipotoxicity is now a well-accepted trigger for metabolic syndrome related NASH [45-48]. In contrast to the levels of saturated free fatty acids (which might induce hepatocyte degeneration via JNK-mediated mitochondrial injury), that often do not differ between NASH and "non-NASH" phenotypes, the levels of biologically reactive free cholesterol (FC) are almost always 
high in NASH compared to benign steatosis [49-52]. In their report, Gan et al. suggest that FC causes hepatocyte apoptotic and necrotic cell death that depends on JNK activation, mitochondrial injury with mitochondrial permeability transition (MPT), ATP depletion, oxidative stress, and caspase-3 activation, but not on ER stress [52]. It is also implicated that JNK1 mediates apoptosis and necrosis and that HMGB1, an archetypical danger-associated molecular pattern (DAMP) molecule, contributes to hepatocellular injury that may be amplified and perpetuated via TLR4 and JNK1 [52]. In another report by Amir et al., JNK 2 has been observed to be hepatoprotective whereas the downstream effector molecule c-Jun causes hepatocyte death by manipulating the cell signaling pathway [53]. In summary, it can be credibly suggested that although the molecular mediators for the pathway are still to be elucidated, oxidative stress is a potent initiator of hepatocyte degeneration or necrosis resulting in the release of DAMPS or alarmins in the microenvironment surrounding the affected cells. Apoptosis has long been regarded as a noninflammatory or even anti-inflammatory mode of cell death, but recent studies suggest that this is not always the case. Necroptosis is a programmed form of necrosis that is engaged under certain conditions when caspase activation is blocked. Necroptosis is also regarded as a highly proinflammatory mode of cell death [54].

\section{Release of ATP: ATP and NAD as DAMPs}

Endogenous molecules and fragments from damaged cells and tissues can be recognized as danger signals, referred to as damage-associated molecular patterns (DAMPs). DAMPs are used for damage-self recognition to evoke immune inflammatory responses and damage healing independent of but in cooperation with exogenous danger signals [55]. In events such as nonspecific cytolysis of healthy cells in physical or stress-related trauma, endogenous molecules such as ATP (3-5 mmoles) may be released extracellularly and can serve as damage-associated molecular patterns (DAMPs) [56]. Increased release of DAMPs can result in cytolysis or necrotic cell death, which generates profound sterile inflammation characterized by accumulation of neutrophils and other immune effector cells [57]. The endogenous or selfmolecules (extracellular matrix proteins (ECM), calciumbinding proteins, and structural proteins) typically function in normal cell homeostasis but are also recognized as danger signals when released into the extracellular space $[58,59]$ exposing hydrophobic portions of the molecules that are normally hidden in healthy living cells [55]. Matzinger and others have extended the danger model as more has been learned about the role of danger signals in tissue injury and other diseases [55, 60-62].

\section{P2X7r Stimulation}

$\mathrm{P} 2 \mathrm{X7r}$ (Purinergic receptor X7) is an atypical member of the purinergic receptor family which when receives a stimulus from a ligand like ATP, an intrinsic ion channel opens up followed by recruitment of a hemichannel protein called pannexin-1. The macrophages which are deficient in $\mathrm{P} 2 \mathrm{X} 7 \mathrm{r}$ have been shown to be unresponsive to ATP as a ligand, corroborating the role of $\mathrm{P} 2 \mathrm{X} 7 \mathrm{r}$ in transmission of the stimulus from such extracellular ligands. It is widely accepted that cytosolic potassium is the key signaling molecule that initiates caspase-1 activation via $\mathrm{K}$ efflux from the cell. Evidence for this pathway is largely based on the ability of elevated extracellular $\mathrm{K}$ to prevent NALP3 inflammasome activation or enzyme activity assays in low K salines [63-65]. Recent data suggest that a second pathway, involving NADPH oxidase, may contribute to caspase-1 activation.

It has also been observed that there is an extracellular ATP-triggered, TNF- $\alpha$ release in microglia, the resident macrophages in the brain, mediated by the P2X7 receptors [66]. Recent reports indicate that the ATP-triggered P2X7 receptor might target NADPH oxidase via extracellular calcium influx, p38 MAPK and PI3 kinase activity, but the sites of these events have only been observed in microgliainduced cortico-neuron injury [67]. The augmentation of P2X7 receptor-induced NADPH oxidase activity has been shown in endotoxin-primed human monocytes [63]. The same study also reported the formation of peroxynitrite from nitric oxide and superoxide released from NADPH oxidase following ATP stimulation in these cells $[63,68]$.

\section{P2X7r in Innate Immunity}

Induction of $\mathrm{P} 2 \mathrm{X} 7 \mathrm{r}$ by ATP can lead to the maturation, chemotaxis, and finally release of TH17 biasing cytokines, IL1 $\beta$, and IL6. Thus, P2X7r has been shown to play a crucial role in the initiation of innate proinflammatory inflammation, DC17 differentiation and Th17-biased immunity [69]. P2X7r's role in a potential modulator of angiogenesis or wound repair has also been reported [70]. Thus, based on the proof available, it would not be wrong to conclude that $\mathrm{P} 2 \mathrm{X} 7 \mathrm{r}$ acts as an innate immune modulator in NASH progression.

\section{P2X7r in Cell Fate in NASH}

Cellular injury plays a central role in triggering inflammation in NAFLD and facilitates its progression to the inflammatory phenotype as seen in NASH and the later fibrotic pathology [71-73]. Normal healthy liver lobules are with little or no accumulation of fat in hepatocytes. On the other hand, stellate cells are laden with fat and start to lose fat when they are in activated state. Normal livers hardly possess activated Kupffer cells (the resident macrophages). NAFLD livers and livers from alcoholic liver injury, CCl4 poisoning, toxin exposure and drug-induced hepatotoxicity show distinct fat accumulation in hepatocytes are largely seen as vacuoles within the cells. Steatosis can be both present in macro- or microvesicular forms. Microsteatosis is likely an epiphenomenon that may be a consequence of acute mitochondrial dysfunction and may not be a direct result of hepatocyte injury. The macrovesicular fat accumulation may or may not be causing lipotoxicity and studies show that triglyceride accumulation can be hepatoprotective [16]. The increased toxicity of the accumulating lipids caused by either the failure of triglyceride synthesis, transport or storage result in intrinsic cellular signaling that end in deciding 


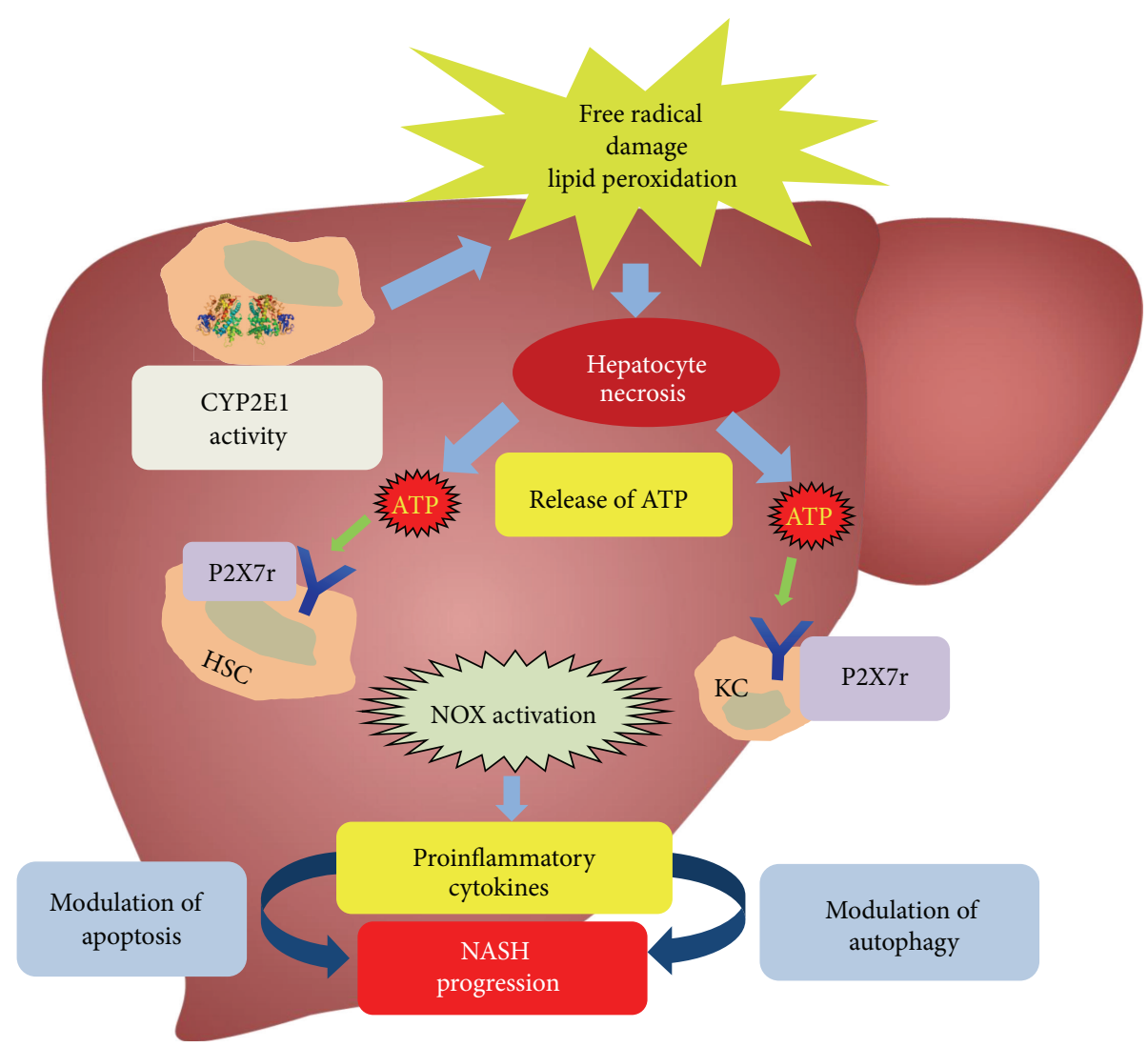

FIGURE 1: Mechanism of CYP2E1-induced oxidative stress and altered cell fate in NASH.

the cellular fate. Many studies report the presence of apoptosis, caspase-independent apoptosis, autophagy, mitophagy, and necrosis. The role of P2X7r has been implicated in the Kupffer cell activation and inflammation, following the release of ATP from necrosed cells in a $\mathrm{CCl}_{4}$-induced model of NASH [74]. According to reports, P2X7r acts as a key regulator in modulating oxidative stress-induced autophagy process [75]. The Das et al. study reports that, when $\mathrm{P} 2 \mathrm{X} 7 \mathrm{r}$ is stimulated by ATP, then it modulates autophagy by depleting LC3B which is an early autophagy marker [75]. At the same time the protein levels of HSC70 and lysosomal membrane association of LAMP2A were marginally upregulated pointing towards chaperone mediated autophagy and release of autophagolysosomes in the extracellular matrix, thus leading to inflammation [76]. Metabolic oxidative stress also, mainly characterized by oxidatively modified proteins, has been known to induce chaperone-mediated autophagy, increased substrate translocation by HSC 70 toward the lysosomal membrane, and increased LAMP2A levels [77]. There exists a correlation between increased lysosomal association of LAMP2A, increase in inflammation and NASH pathophysiology $[75,78,79]$. Das et al. did not report LAMP2A depletion, whereas there was an earlier report by Fortunato and Kroemer emphasizing the depletion of LAMP2A that also stated that impaired lysosomal membrane association and relative paucity of autolysosomes reduced ATP levels and finally resulted in a shift from apoptosis to necrosis [80]. Since defective autophagy results in depletion of LC3B, and a decrease in LC3B levels has been already proven to be a driver of caspase 1 activation, thus indicating a shift towards apoptosis and inflammation, it can be well assumed that P2X7r acts as molecular switch crucial for deciding the cell fate also the programmed pathway of cell survival/death in $\mathrm{NASH}$, before it finally moves on to necrotic damage [81].

\section{Conclusions}

As an ion channel protein that is responsive to ATP and NAD at the cellular membrane, $\mathrm{P} 2 \mathrm{X} 7$ receptor might play a key role in modulating the cell fate in NASH. As described in the review, oxidative stress either in the hepatocytes themselves due to lipotoxicity or metabolic disturbances because abnormal fat storage can trigger the release of damage associated molecular patterns into the highly mobile extracellular space that can activate nearby resident macrophages, endothelial cells, or stellate cells. Based on the recent findings that P2X7 receptors are located on the membranes of all the cell types of the liver sinusoidal area, it may be assumed that the ion channel protein will be activated on these cells [75]. Further, these receptors can trigger the activation of NADPH oxidase isoforms, especially NOX2 and NOX4 in the sinusoidal cells to cause their activation and result in uninterrupted innate immune signaling, release of proinflammatory cytokines. Since the proinflammatory events have been correlated well with the cellular longevity and fate in the liver microenvironment, it is justifiable to assume that depending on 
the nature of insult the liver cells will undergo apoptosis, autophagy, or necrosis. The chain of events that P2X7 receptor stimulation may trigger and a detailed understanding of its pathway that covers oxidative stress-innate immune crosstalk may serve as a crucial benchmark in understanding NASH pathophysiology and help design new therapeutic strategies for this metabolic disease (Figure 1).

\section{Conflict of Interests}

The authors declare that there is no conflict of interests regarding the publication of this paper.

\section{Acknowledgments}

The authors thank Dr. Anna Mae Diehl, Division of Gastroenterology, Duke University, for careful editing and adding valuable insights to the paper. The work is supported by NIH grants R00ES19875 and P01AT039641 to Saurabh Chatterjee. This work has been supported by NIH Pathway to Independence Award, R00ES019875.

\section{References}

[1] G. S. Hotamisligil, N. S. Shargill, and B. M. Spiegelman, "Adipose expression of tumor necrosis factor- $\alpha$ : direct role in obesitylinked insulin resistance," Science, vol. 259, no. 5091, pp. 87-91, 1993.

[2] G. Reaven, "Metabolic syndrome: pathophysiology and implications for management of cardiovascular disease," Circulation, vol. 106, no. 3, pp. 286-288, 2002.

[3] E. J. Gallagher, D. Leroith, and E. Karnieli, "Insulin resistance in obesity as the underlying cause for the metabolic syndrome," Mount Sinai Journal of Medicine, vol. 77, no. 5, pp. 511-523, 2010.

[4] S. H. Chen, F. He, H. L. Zhou, H. R. Wu, C. Xia, and Y. M. Li, "Relationship between nonalcoholic fatty liver disease and metabolic syndrome," Journal of Digestive Diseases, vol. 12, no. 2, pp. 125-130, 2011.

[5] C. E. Kelley, A. J. Brown, A. M. Diehl, and T. L. Setji, "Review of nonalcoholic fatty liver disease in women with polycystic ovary syndrome," World Journal of Gastroenterology, vol. 20, no. 39, pp. 14172-14184, 2014.

[6] N. Chalasani, Z. Younossi, J. E. Lavine et al., "The diagnosis and management of non-alcoholic fatty liver disease: practice Guideline by the American Association for the Study of Liver Diseases, American College of Gastroenterology, and the American Gastroenterological Association," Hepatology, vol. 55, no. 6, pp. 2005-2023, 2012.

[7] P. Angulo, "Treatment of nonalcoholic fatty liver disease," Annals of Hepatology, vol. 1, no. 1, pp. 12-19, 2002.

[8] G. Vernon, A. Baranova, and Z. M. Younossi, "Systematic review: the epidemiology and natural history of non-alcoholic fatty liver disease and non-alcoholic steatohepatitis in adults," Alimentary Pharmacology \& Therapeutics, vol. 34, no. 3, pp. 274-285, 2011.

[9] B. N. Bohinc and A. M. Diehl, "Mechanisms of disease progression in NASH: new paradigms," Clinics in Liver Disease, vol. 16, no. 3, pp. 549-565, 2012.

[10] N. Rosso, N. C. Chavez-Tapia, C. Tiribelli, and S. Bellentani, "Translational approaches: from fatty liver to non-alcoholic steatohepatitis," World Journal of Gastroenterology, vol. 20, no. 27, pp. 9038-9049, 2014.

[11] A. M. Diehl, "Hepatic complications of obesity," Gastroenterology Clinics of North America, vol. 39, no. 1, pp. 57-68, 2010.

[12] M. Pasarín, V. La Mura, J. Gracia-Sancho et al., "Sinusoidal endothelial dysfunction precedes inflammation and fibrosis in a model of NAFLD," PLoS ONE, vol. 7, no. 4, Article ID e32785, 2012.

[13] C. P. Day and O. F. W. James, "Steatohepatitis: a tale of two "Hits"?" Gastroenterology, vol. 114, no. 4, pp. 842-845, 1998.

[14] S. Q. Yang, H. Z. Lin, M. D. Lane, M. Clemens, and A. M. Diehl, "Obesity increases sensitivity to endotoxin liver injury: implications for the pathogenesis of steatohepatitis," Proceedings of the National Academy of Sciences of the United States of America, vol. 94, no. 6, pp. 2557-2562, 1997.

[15] K. Yamaguchi, L. Yang, S. McCall et al., "Inhibiting triglyceride synthesis improves hepatic steatosis but exacerbates liver damage and fibrosis in obese mice with nonalcoholic steatohepatitis," Hepatology, vol. 45, no. 6, pp. 1366-1374, 2007.

[16] S. S. Choi and A. M. Diehl, "Hepatic triglyceride synthesis and nonalcoholic fatty liver disease," Current Opinion in Lipidology, vol. 19, no. 3, pp. 295-300, 2008.

[17] M. F. Abdelmalek and A. M. Diehl, "Nonalcoholic fatty liver disease as a complication of insulin resistance," The Medical Clinics of North America, vol. 91, no. 6, pp. 1125-1149, 2007.

[18] E. Bugianesi, S. Moscatiello, M. F. Ciaravella, and G. Marchesini, "Insulin resistance in nonalcoholic fatty liver disease," Current Pharmaceutical Design, vol. 16, no. 17, pp. 1941-1951, 2010.

[19] E. Bugianesi, C. Zannoni, E. Vanni, R. Marzocchi, and G. Marchesini, "Non-alcoholic fatty liver and insulin resistance: a cause-effect relationship?" Digestive and Liver Disease, vol. 36, no. 3, pp. 165-173, 2004.

[20] H. Tilg and A. R. Moschen, "Evolution of inflammation in nonalcoholic fatty liver disease: the multiple parallel hits hypothesis," Hepatology, vol. 52, no. 5, pp. 1836-1846, 2010.

[21] Y. Sumida, E. Niki, Y. Naito, and T. Yoshikawa, "Involvement of free radicals and oxidative stress in NAFLD/NASH," Free Radical Research, vol. 47, no. 11, pp. 869-880, 2013.

[22] S. Gawrieh, E. C. Opara, and T. R. Koch, "Oxidative stress in nonalcoholic fatty liver disease: pathogenesis and antioxidant therapies," Journal of Investigative Medicine, vol. 52, no. 8, pp. 506-514, 2004.

[23] L. A. Videla, R. Rodrigo, J. Araya, and J. Poniachik, "Insulin resistance and oxidative stress interdependency in nonalcoholic fatty liver disease," Trends in Molecular Medicine, vol. 12, no. 12, pp. 555-558, 2006.

[24] L. A. Videla, R. Rodrigo, J. Araya, and J. Poniachik, “Oxidative stress and depletion of hepatic long-chain polyunsaturated fatty acids may contribute to nonalcoholic fatty liver disease," Free Radical Biology and Medicine, vol. 37, no. 9, pp. 1499-1507, 2004.

[25] J. Aubert, K. Begriche, L. Knockaert, M. A. Robin, and B. Fromenty, "Increased expression of cytochrome P450 2E1 in nonalcoholic fatty liver disease: mechanisms and pathophysiological role," Clinics and Research in Hepatology and Gastroenterology, vol. 35, no. 10, pp. 630-637, 2011.

[26] M. Orellana, R. Rodrigo, N. Varela et al., "Relationship between in vivo chlorzoxazone hydroxylation, hepatic cytochrome P450 $2 \mathrm{E} 1$ content and liver injury in obese non-alcoholic fatty liver disease patients," Hepatology Research, vol. 34, no. 1, pp. 57-63, 2006. 
[27] E. Niki, "Lipid peroxidation: physiological levels and dual biological effects," Free Radical Biology \& Medicine, vol. 47, no. 5, pp. 469-484, 2009.

[28] L. Malaguarnera, R. Madeddu, E. Palio, N. Arena, and M. Malaguarnera, "Heme oxygenase-1 levels and oxidative stressrelated parameters in non-alcoholic fatty liver disease patients," Journal of Hepatology, vol. 42, no. 4, pp. 585-591, 2005.

[29] S. Sutti, A. Jindal, I. Locatelli et al., "Adaptive immune responses triggered by oxidative stress contribute to hepatic inflammation in NASH," Hepatology, vol. 59, no. 3, pp. 886-897, 2014.

[30] S. Seki, T. Kitada, T. Yamada, H. Sakaguchi, K. Nakatani, and K. Wakasa, "In situ detection of lipid peroxidation and oxidative DNA damage in non-alcoholic fatty liver diseases," Journal of Hepatology, vol. 37, no. 1, pp. 56-62, 2002.

[31] N. Chalasani, M. A. Deeg, and D. W. Crabb, "Systemic levels of lipid peroxidation and its metabolic and dietary correlates in patients with nonalcoholic steatohepatitis," The American Journal of Gastroenterology, vol. 99, no. 8, pp. 1497-1502, 2004.

[32] Y. I. Miller, S.-H. Choi, P. Wiesner et al., "Oxidation-specific epitopes are danger-associated molecular patterns recognized by pattern recognition receptors of innate immunity," Circulation Research, vol. 108, no. 2, pp. 235-248, 2011.

[33] D. Weismann and C. J. Binder, "The innate immune response to products of phospholipid peroxidation," Biochimica et Biophysica Acta, vol. 1818, no. 10, pp. 2465-2475, 2012.

[34] Z. Tariq, C. J. Green, and L. Hodson, "Are oxidative stress mechanisms the common denominator in the progression from hepatic steatosis towards non-alcoholic steatohepatitis (NASH)?” Liver International, vol. 34, no. 7, pp. e180-e190, 2014.

[35] A. Takaki, D. Kawai, and K. Yamamoto, "Multiple hits, including oxidative stress, as pathogenesis and treatment target in non-alcoholic steatohepatitis (NASH)," International Journal of Molecular Sciences, vol. 14, no. 10, pp. 20704-20728, 2013.

[36] E. Novo, C. Busletta, L. V. D. Bonzo et al., "Intracellular reactive oxygen species are required for directional migration of resident and bone marrow-derived hepatic pro-fibrogenic cells," Journal of Hepatology, vol. 54, no. 5, pp. 964-974, 2011.

[37] E. Novo, D. Povero, C. Busletta et al., "The biphasic nature of hypoxia-induced directional migration of activated human hepatic stellate cells," The Journal of Pathology, vol. 226, no. 4, pp. 588-597, 2012.

[38] S. Chatterjee, D. Ganini, E. J. Tokar et al., "Leptin is key to peroxynitrite-mediated oxidative stress and Kupffer cell activation in experimental non-alcoholic steatohepatitis," Journal of Hepatology, vol. 58, no. 4, pp. 778-784, 2013.

[39] J. Zielonka, A. Sikora, J. Joseph, and B. Kalyanaraman, "Peroxynitrite is the major species formed from different flux ratios of co-generated nitric oxide and superoxide: direct reaction with boronate-based fluorescent probe," The Journal of Biological Chemistry, vol. 285, no. 19, pp. 14210-14216, 2010.

[40] M. J. Czaja, "Cell signaling in oxidative stress-induced liver injury," Seminars in Liver Disease, vol. 27, no. 4, pp. 378-389, 2007.

[41] R. Singh, Y. Wang, J. M. Schattenberg, Y. Xiang, and M. J. Czaja, "Chronic oxidative stress sensitizes hepatocytes to death from 4-hydroxynonenal by JNK/c-Jun overactivation," American Journal of Physiology: Gastrointestinal and Liver Physiology, vol. 297, no. 5, pp. G907-G917, 2009.

[42] M. J. Czaja, H. Liu, and Y. Wang, "Oxidant-induced hepatocyte injury from menadione is regulated by ERK and AP-1 signaling," Hepatology, vol. 37, no. 6, pp. 1405-1413, 2003.
[43] S. R. Bruckner and S. Estus, "JNK3 contributes to c-jun induction and apoptosis in 4-hydroxynonenal-treated sympathetic neurons," Journal of Neuroscience Research, vol. 70, no. 5, pp. 665-670, 2002.

[44] M. Parola, G. Robino, F. Marra et al., "HNE interacts directly with JNK isoforms in human hepatic stellate cells," The Journal of Clinical Investigation, vol. 102, no. 11, pp. 1942-1950, 1998.

[45] B. A. Neuschwander-Tetri, "Nontriglyceride hepatic lipotoxicity: the new paradigm for the pathogenesis of NASH," Current Gastroenterology Reports, vol. 12, no. 1, pp. 49-56, 2010.

[46] B. A. Neuschwander-Tetri, "Hepatic lipotoxicity and the pathogenesis of nonalcoholic steatohepatitis: the central role of nontriglyceride fatty acid metabolites," Hepatology, vol. 52, no. 2, pp. 774-788, 2010.

[47] K. Cusi, "Role of obesity and lipotoxicity in the development of nonalcoholic steatohepatitis: pathophysiology and clinical implications," Gastroenterology, vol. 142, no. 4, pp. 711.e6725.e6, 2012.

[48] G. Farrell, "Should we lower lipids in nonalcoholic fatty liver disease?" Clinical Gastroenterology and Hepatology, vol. 12, no. 1, pp. 152-155, 2014.

[49] P. Puri, R. A. Baillie, M. M. Wiest et al., "A lipidomic analysis of nonalcoholic fatty liver disease," Hepatology, vol. 46, no. 4, pp. 1081-1090, 2007.

[50] D. M. van Rooyen, C. Z. Larter, W. G. Haigh et al., "Hepatic free cholesterol accumulates in obese, diabetic mice and causes nonalcoholic steatohepatitis," Gastroenterology, vol. 141, no. 4, pp. 1393.e5-1403.e5, 2011.

[51] F. Caballero, A. Fernández, A. M. De Lacy, J. C. FernándezCheca, J. Caballería, and C. García-Ruiz, "Enhanced free cholesterol, SREBP-2 and StAR expression in human NASH," Journal of Hepatology, vol. 50, no. 4, pp. 789-796, 2009.

[52] L. T. Gan, D. M. Van Rooyen, M. E. Koina, R. S. McCuskey, N. C. Teoh, and G. C. Farrell, "Hepatocyte free cholesterol lipotoxicity results from JNK1-mediated mitochondrial injury and is HMGB1 and TLR4-dependent," Journal of Hepatology, vol. 61, no. 6, pp. 1376-1384, 2014.

[53] M. Amir, K. Liu, E. Zhao, and M. J. Czaja, "Distinct functions of JNK and c-Jun in oxidant-induced hepatocyte death," Journal of Cellular Biochemistry, vol. 113, no. 10, pp. 3254-3265, 2012.

[54] P. Davidovich, C. J. Kearney, and S. J. Martin, "Inflammatory outcomes of apoptosis, necrosis and necroptosis," Biological Chemistry, vol. 395, no. 10, pp. 1163-1171, 2014.

[55] P. Matzinger, "Friendly and dangerous signals: is the tissue in control?” Nature Immunology, vol. 8, no. 1, pp. 11-13, 2007.

[56] M. E. Bianchi, "DAMPs, PAMPs and alarmins: all we need to know about danger," Journal of Leukocyte Biology, vol. 81, no. 1, pp. 1-5, 2007.

[57] D. Yang, G. de la Rosa, P. Tewary, and J. J. Oppenheim, "Alarmins link neutrophils and dendritic cells," Trends in Immunology, vol. 30, no. 11, pp. 531-537, 2009.

[58] D. L. Rosin and M. D. Okusa, "Dangers within: DAMP responses to damage and cell death in kidney disease," Journal of the American Society of Nephrology, vol. 22, no. 3, pp. 416-425, 2011.

[59] H. Kono and K. L. Rock, "How dying cells alert the immune system to danger," Nature Reviews Immunology, vol. 8, no. 4, pp. 279-289, 2008.

[60] P. Matzinger, "An innate sense of danger," Annals of the New York Academy of Sciences, vol. 961, pp. 341-342, 2002. 
[61] S.-Y. Seong and P. Matzinger, "Hydrophobicity: an ancient damage-associated molecular pattern that initiates innate immune responses," Nature Reviews Immunology, vol. 4, no. 6, pp. 469-478, 2004.

[62] M. Xiang and J. Fan, "Pattern recognition receptor-dependent mechanisms of acute lung injury," Molecular Medicine, vol. 16, no. 1-2, pp. 68-82, 2010.

[63] J. Hewinson, S. F. Moore, C. Glover, A. G. Watts, and A. B. MacKenzie, "A key role for redox signaling in rapid P2X7 receptor-induced IL-1 $\beta$ processing in human monocytes," Journal of Immunology, vol. 180, no. 12, pp. 8410-8420, 2008.

[64] J. M. Kahlenberg and G. R. Dubyak, "Mechanisms of caspase-1 activation by $\mathrm{P} 2 \mathrm{X} 7$ receptor-mediated K+ release," The American Journal of Physiology-Cell Physiology, vol. 286, no. 5, pp. C1100-C1108, 2004.

[65] V. Pétrilli, S. Papin, C. Dostert, A. Mayor, F. Martinon, and J. Tschopp, "Activation of the NALP3 inflammasome is triggered by low intracellular potassium concentration," Cell Death and Differentiation, vol. 14, no. 9, pp. 1583-1589, 2007.

[66] T. Suzuki, I. Hide, K. Ido, S. Kohsaka, K. Inoue, and Y. Nakata, "Production and release of neuroprotective tumor necrosis factor by P2X7 receptor-activated microglia," The Journal of Neuroscience, vol. 24, no. 1, pp. 1-7, 2004.

[67] L. K. Parvathenani, S. Tertyshnikova, C. R. Greco, S. B. Roberts, B. Robertson, and R. Posmantur, "P2X7 mediates superoxide production in primary microglia and is up-regulated in a transgenic mouse model of Alzheimer's disease," The Journal of Biological Chemistry, vol. 278, no. 15, pp. 13309-13317, 2003.

[68] X. Wang, P. Svedin, C. Nie et al., "N-Acetylcysteine reduces lipopolysaccharide-sensitized hypoxic-ischemic brain injury," Annals of Neurology, vol. 61, no. 3, pp. 263-271, 2007.

[69] M. E. Killeen, L. Ferris, E. A. Kupetsky, L. Falo Jr., and A. R. Mathers, "Signaling through purinergic receptors for ATP induces human cutaneous innate and adaptive Th17 responses: implications in the pathogenesis of psoriasis," Journal of Immunology, vol. 190, no. 8, pp. 4324-4336, 2013.

[70] L. M. Hill, M. L. Gavala, L. Y. Lenertz, and P. J. Bertics, "Extracellular ATP may contribute to tissue repair by rapidly stimulating purinergic receptor X7-dependent vascular endothelial growth factor release from primary human monocytes," Journal of Immunology, vol. 185, no. 5, pp. 3028-3034, 2010.

[71] S. Choi and A. M. Diehl, "Role of inflammation in nonalcoholic steatohepatitis," Current Opinion in Gastroenterology, vol. 21, no. 6, pp. 702-707, 2005.

[72] A. M. Diehl, Z. P. Li, H. Z. Lin, and S. Q. Yang, "Cytokines and the pathogenesis of non-alcoholic steatohepatitis," Gut, vol. 54, no. 2, pp. 303-306, 2005.

[73] H. Tilg and A. M. Diehl, "Cytokines in alcoholic and nonalcoholic steatohepatitis," The New England Journal of Medicine, vol. 343, no. 20, pp. 1467-1467, 2000.

[74] S. Chatterjee, R. Rana, J. Corbett, M. B. Kadiiska, J. Goldstein, and R. P. Mason, "P2X7 receptor-NADPH oxidase axis mediates protein radical formation and Kupffer cell activation in carbon tetrachloride-mediated steatohepatitis in obese mice," Free Radical Biology and Medicine, vol. 52, no. 9, pp. 1666-1679, 2012.

[75] S. Das, R. K. Seth, A. Kumar et al., "Purinergic receptor X7 is a key modulator of metabolic oxidative stress-mediated autophagy and inflammation in experimental nonalcoholic steatohepatitis," The American Journal of Physiology-Gastrointestinal and Liver Physiology, vol. 305, no. 12, pp. G950-G963, 2013.
[76] T. Takenouchi, M. Nakai, Y. Iwamaru et al., "The activation of $\mathrm{P} 2 \mathrm{X} 7$ receptor impairs lysosomal functions and stimulates the release of autophagolysosomes in microglial cells," Journal of Immunology, vol. 182, no. 4, pp. 2051-2062, 2009.

[77] R. Kiffin, C. Christian, E. Knecht, and A. M. Cuervo, "Activation of chaperone-mediated autophagy during oxidative stress," Molecular Biology of the Cell, vol. 15, no. 11, pp. 4829-4840, 2004.

[78] S. Das, A. Kumar, R. K. Seth et al., "Proinflammatory adipokine leptin mediates disinfection byproduct bromodichloromethane-induced early steatohepatitic injury in obesity," Toxicology and Applied Pharmacology, vol. 269, no. 3, pp. 297-306, 2013.

[79] V. Deretic, "Autophagy as an innate immunity paradigm: expanding the scope and repertoire of pattern recognition receptors," Current Opinion in Immunology, vol. 24, no. 1, pp. 21-31, 2012.

[80] F. Fortunato and G. Kroemer, "Impaired autophagosomelysosome fusion in the pathogenesis of pancreatitis," Autophagy, vol. 5, no. 6, pp. 850-853, 2009.

[81] K. Nakahira, J. A. Haspel, V. A. K. Rathinam et al., "Autophagy proteins regulate innate immune responses by inhibiting the release of mitochondrial DNA mediated by the NALP3 inflammasome," Nature Immunology, vol. 12, no. 3, pp. 222-230, 2011. 


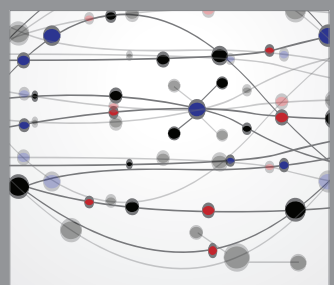

The Scientific World Journal
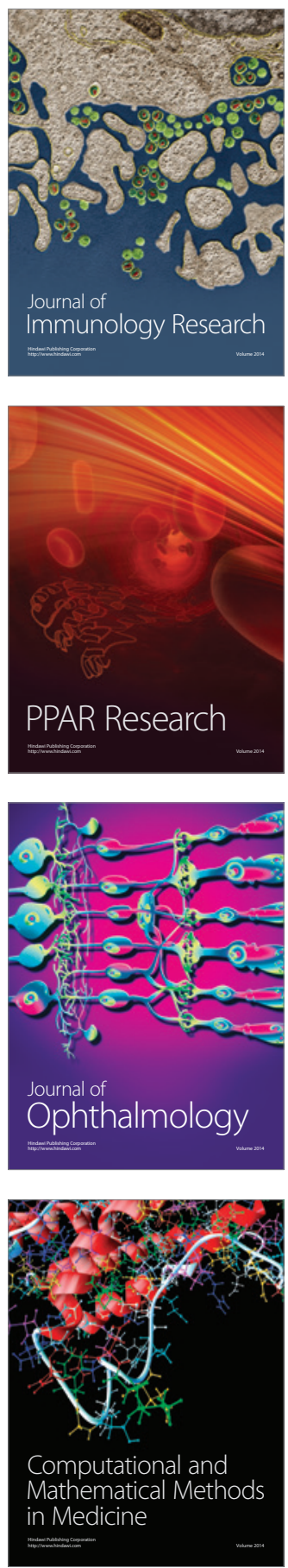

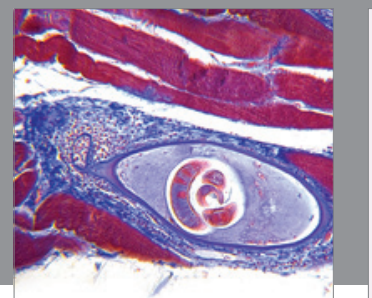

Gastroenterology

Research and Practice
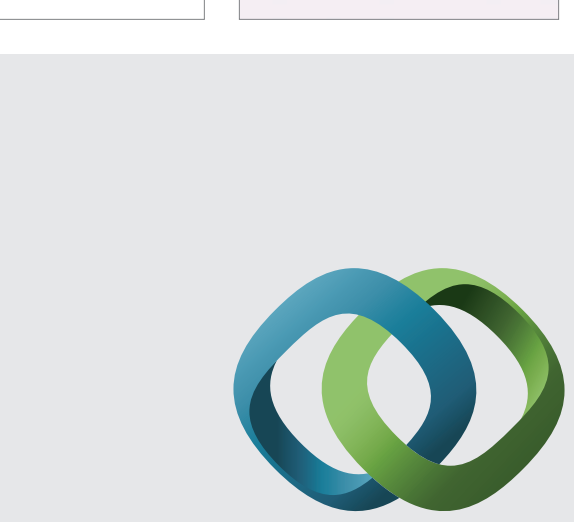

\section{Hindawi}

Submit your manuscripts at

http://www.hindawi.com
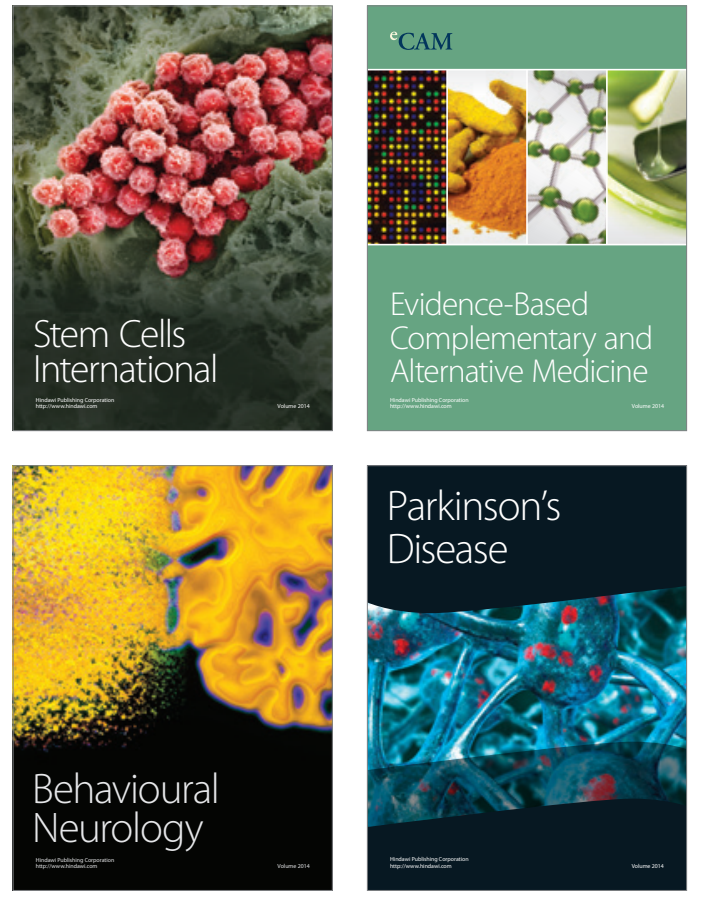
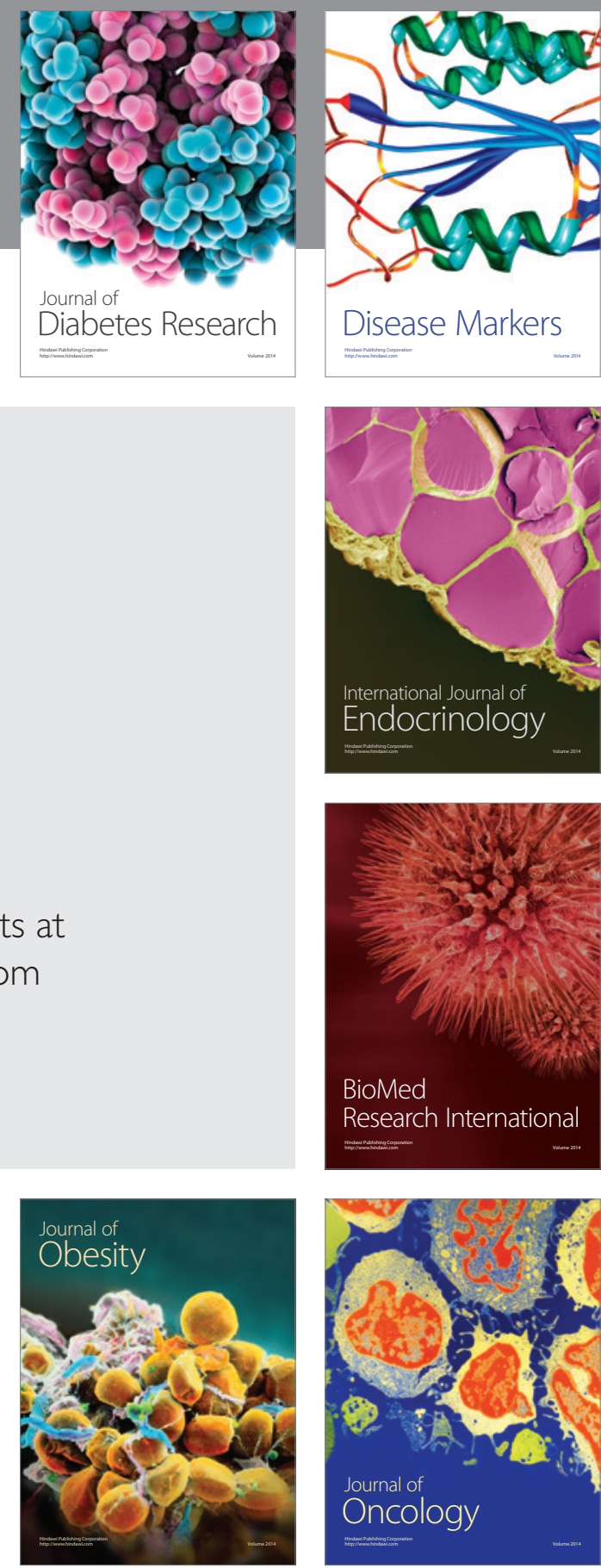

Disease Markers
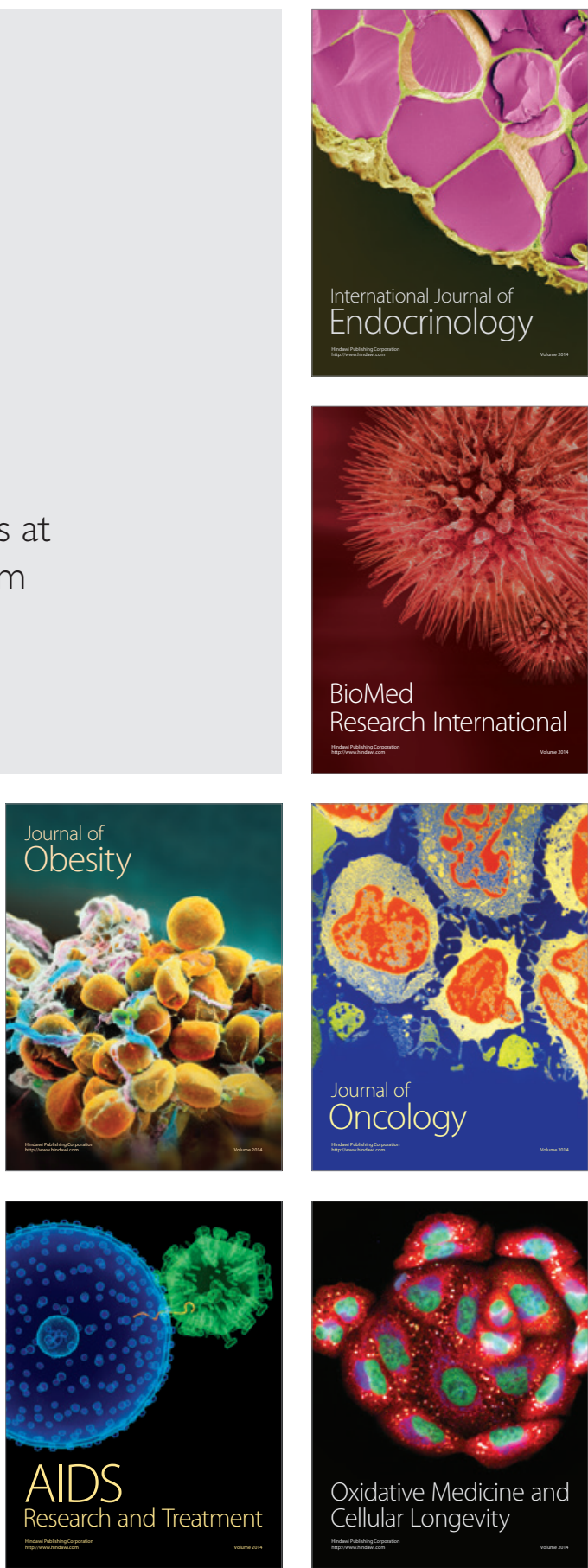